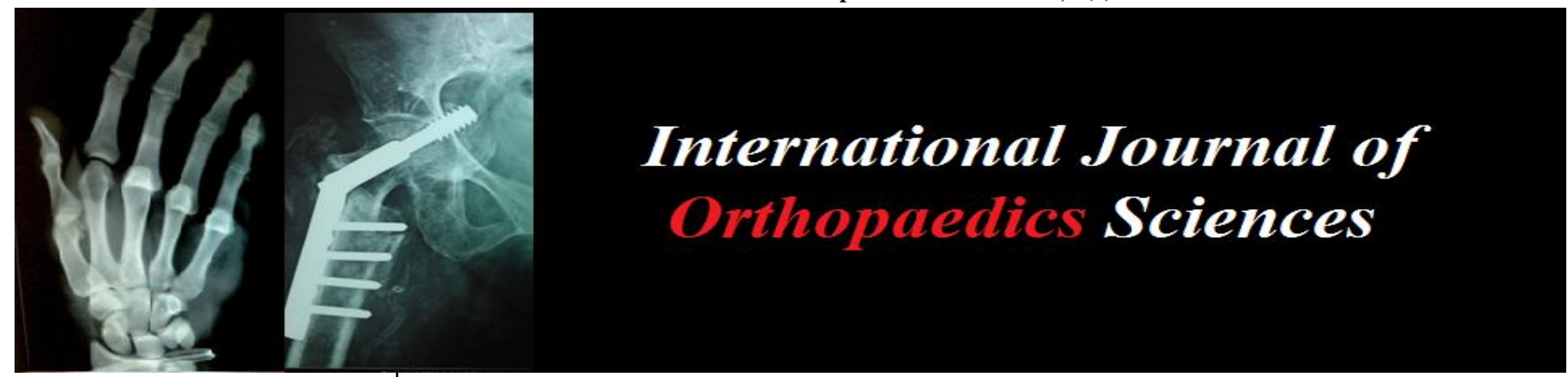

ISSN: $2395-1958$

IJOS 2017; 3(4): 160-164

(C) 2017 IJOS

www.orthopaper.com

Received: 05-08-2017

Accepted: 06-09-2017

Dr. Atul Agrawal

MS Orthopaedics, Professor, Department of Orthopaedics,

Himalayan Institute of Medical

Sciences, Swami Rama

Himalayan University, Jolly Grant, Dehradun, Uttarakhand, India

Dr. Faiz Akbar Siddiqui MS Orthopaedics, Senior Resident, Department of Orthopaedics, Himalayan Institute of Medical Sciences, Swami Rama Himalayan University, Jolly Grant, Dehradun, Uttarakhand, India

Dr. Ruchit Khera

Assistant Professor, Department of Orthopaedics, Himalayan

Institute of Medical Sciences,

Swami Rama Himalayan

University, Jolly Grant,

Dehradun, Uttarakhand, India

Dr. Amish Bhandari

Senior Resident, Department of Orthopaedics, Himalayan

Institute of Medical Sciences,

Swami Rama Himalayan

University, Jolly Grant,

Dehradun, Uttarakhand, India

Correspondence

Dr. Atul Agrawa

MS Orthopaedics, Professor,

Department of Orthopaedics,

Himalayan Institute of Medical

Sciences, Swami Rama

Himalayan University, Jolly

Grant, Dehradun, Uttarakhand,

India

\section{Results with primary repair of the avulsed anterior cruciate ligament and internal fiber ligament augmentation}

\author{
Dr. Atul Agrawal, Dr. Faiz Akbar Siddiqui, Dr. Ruchit Khera and \\ Dr. Amish Bhandari
}

DOI: $\underline{\text { https://doi.org/10.22271/ortho.2017.v3.i4c.23 }}$

\section{Abstract}

Introduction: ACL repair was first performed over a century ago, but reconstruction has overwhelmingly been the surgery of choice for operative ACL ruptures since the 1970s. Modern arthroscopic surgical instrumentation has made repair of ACL tissue easier and advancements in functional tissue engineering and regenerative medicine have resulted in a renewed interest in ACL repair. This is an attractive option to restore normal patient anatomy, retaining proprioceptive fibers, and not causing donor site morbidity that can be associated with reconstructions.

Material \& Methods: The study included 10 consecutive patients who presented with traumatic ACL avulsions (including bony avulsions) in outpatient or emergency department in last 2 years. Our inclusion criteria were cases with primary ACL injury (avulsion / proximal or distal end) not exceeding 3 months. Patients with severe arthritis of knee, multiligamantous ligament injury, history of previous surgery, bleeding disorders, any comorbidility leading to a non-operable condition were excluded from the study. Results: Out of 10 cases three cases had an avulsion from femoral end while seven had it from the tibial end. The injury surgey interval was in a range of six days to sixtythree days. Post operatively four patients had restriction of knee extension (5 degrees) at 4 weeks follow up. Rest of the cases had attained full range of motion at four weeks. All the cases had pain free gait pattern but needed aggressive physiotherapy to reinforce the normal gait pattern. There was no clinical ACL laxity in any of the cases, whenever seen at routine follow ups

Conclusion: The internal brace acts as secondary pillar which supports the strength of the repaired ligament. Patients have a better proprioception, faster recovery to work \& are safe guarded against an unpredictable outcome of ACL reconstruction.

Keywords: Primary ACL Repair, Internal Fiber ligament bracing, Avulsion injuries

\section{Introduction}

The anterior cruciate ligament (ACL) is one of the most studied \& commonly injured ligaments in the body. The research concerning its inherent healing and surgical repair dates back to the late 19 th century ${ }^{[1-5]}$.

ACL repair was first performed over a century ago, but reconstruction has overwhelmingly been the surgery of choice for operative ACL ruptures since the 1970s [1-5]. Mayo-Robson ${ }^{[6]}$, performed the first successful ACL repair on a coal miner in 1895. In his 1903 publication, he described a simple suturing of the patients torn ACL and posterior cruciate ligament (PCL), achieving a favorable outcome in which the patient returned to work with a stable knee.In a randomised controlled trial in 1987, Sandberg showed that there was no difference in outcomes after early primary repair versus conservative treatment ${ }^{[7]}$. ACL repair techniques are re-emerging as a promising treatment option for acute proximal ruptures. Repair of the ACL can be performed successfully and has the advantage of retaining the natural proprioceptive fibres of the ligament. The internal brace acts as a secondary stabiliser after repair, which may allow accelerated rehabilitation and return to sports, whilst resisting injury recurrence when this is possible.

In 1991, Sherman et al. ${ }^{[8]}$ established the first classification for types of ACL tear based upon anatomic location. 


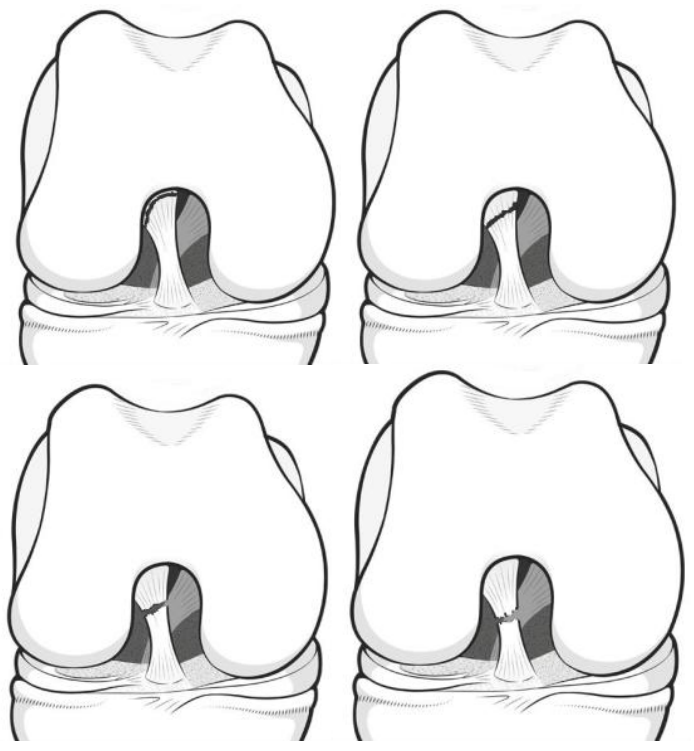

Fig 1: Sherman classification of ACL tears based upon anatomical location. (Type I is where the ligament literally pulls right off the bone from where it takes its origin in the notch of the femur. Type II is when the tear is close to, but not quite at, the attachment point on the wall of the femur. Type III is about a third of the way along the ligament. Type IV is a mid-substance tear, right in the middle of the ligament.)

Anterior cruciate ligament (ACL) avulsion fractures or tibial eminence avulsion fractures are a type of avulsion fracture of the knee. This typically involves separation of the tibial attachment of the ACL to variable degrees. Separation at the femoral attachment is rare ${ }^{[9]}$.

Meyers and McKeevers classification - This classification was initially proposed by Meyers and McKeevers in 1959, and later has been modified by Zaricznyj in $1977^{[10]}$, which remains the most widely used system of describing Anterior cruciate ligament avulsion fracture. They are classified into four main types under the Meyers and McKeever system: Type 1: minimally/nondisplaced fragment; Type 2: anterior elevation of the fragment; Type 3: complete separation of the fragment; Type 3a: involves small portion of eminence; Type 3b: involves the majority of the eminence; Type 4: comminuted avulsion or a rotation of the fracture fragment. Failure rates were postulated to be higher than $40 \%$ after early attempts at ACL surgical repair. Unsatisfactory outcomes led to abandonment of the primary repair technique in favor of ACL reconstruction. Autologous hamstring graft of gracilis \& semitendonosus or patella tendon graft started being harvested to replace the ACL by removing all the remnants\& clearing the debris of the injured ligament.

However, ACL reconstruction has been associated with a number of problems. Firstly, a degree of morbidity is associated with auto grafts, with hamstring muscle weakness following harvesting, and anterior knee pain common with patellar tendon grafts. Secondly, reconstruction with graft material does not restore the proprioceptive properties that the native ligament possesses, which possibly explains why some athletes never regain the same level of sporting performance as pre-injury, even after reconstruction. Thirdly, and possibly subsequently, long-term follow-up data shows high rates of post traumatic osteoarthritis within 20 years, despite treatment with ACL reconstruction. Modern arthroscopic surgical instrumentation has made repair of ACL tissue easier and advancements in functional tissue engineering and regenerative medicine have resulted in a renewed interest in
ACL repair. This is an attractive option to restore normal patient anatomy, retaining proprioceptive fibres, and not causing donor site morbidity that can be associated with reconstructions.

Hence in this study we aim to describe this noval technique to repair an avulsed ACL ligament along with fiber ligament bracing and to assess the post-operative outcome of such cases.

\section{Material \& Methods}

This study was conducted in the department of orthopaedics, Himalayan Institute of medical sciences, Swami Rama Himalayan University, Dehradun. The study included 10 consecutive patients who presented with traumatic acl avulsions (including bony avulsions) in outpatient or emergency department in last 2 years. Our inclusion criteria were cases with primary ACL injury (avulsion / proximal or distal end) not exceeding 3 months. Patients with severe arthritis of knee, multiligamantous ligament injury, history of previous surgery, bleeding disorders, any comorbidility leading to a non-operable condition were excluded from the study. All the patients who were candidates to be enrolled for the study were screened in detail with clinical examination using anterior drawer test, lachman test, Pivot shift test, Posterior drawer test and mcmurray test. The potential cases for the enrolment were investigated with MRI of the injured knee to assess the nature of injury and to rule out any coexistant Ligamentous injury. After all the inclusion \& exclusion workup, clinical and investigational assessment and taking a written informed consent we were able to recruit 10 patients for enrolment in the study. All patients were explained with a written consent that in an event that on intraoperative assessment ACL quality was poor or irreparably damaged then they would be opted out of the study and a standard ACL reconstruction would be performed. Our ideal cases for the study would be those with intraoperative broad ACL stump with mild interstitial tearing and the ability to hold sutures, ${ }^{[11]}$. Post operatively patients were kept on Knee Range of Motion (ROM) brace locked in 5 degree short of full extension. The brace was continued for 4 weeks. Intermittent knee ROM exercises were started after suture removal but not allowed to bend the knee more than 90 degree till 3 weeks post operatively. Patients were kept nonweight bearing for 4 weeks. Non-weight bearing/ toe touch mobilization was started from weeks post operatively. Physiotherapy for the quadriceps and hamstring were started as soon as the pain was tolerable to start physiotherapy.

\section{Surgical Technique}

The ACL repair technique involved repair of the ligament where it had avulsed from its femoral attachment on the medial wall of the lateral femoral condyle or its tibial origin from the plateau. The ACL remnant was "whip stitched" using an arthroscopic suture passing instrument. The proximal end in femoral avulsions \& distal end in tibial avulsions of the ACL was then re-approximated against the medial wall of the lateral femoral condyle in femoral avulsions \&tibial plateau in tibial avulsions, in an anatomical mid-bundle position. The bone on the femoral \& tibial end at the anatomical insertion $\&$ origin point was freshened with a micro-fracture probe. The repair was then protected using the Internal- Brace. Ligament Augmentation Repair device, a $2.0 \mathrm{~mm}$ braided ultrahigh molecular weight polyethylene tape bridging from tibia to femur. Tensioning of the repaired ligament was carried out with the knee in extension. To facilitate the repair - and for 
fixation- $4.0 \mathrm{~mm}$ tunnel was drilled in the femur / tibia as the case may be. Fixation of the repaired ligament was carried out by passing the fiber tape through the femoral/ tibial insertion tunnel \& further tensioning the ligament in extension the fiber tape was locked with a suture dish. Post operatively; the patient underwent the standard ACL rehabilitation program from our institute, which consists of early-accelerated recovery and protected weight bearing with brace in situ.

\section{Results}

The results obtained were meticulously recorded and analysed to draw conclusions from the study. Total of 10 cases were enrolled in the study over a period of two years (Table I). There were eight males and two females in the study. All patients were between 17 to 38 year old. All the cases had come with complaints of Pain in effected knee with difficulty in walking $\&$ had a common cause of injury pattern subjecting to road traffic accident (RTA). All Patients were otherwise fit and well and an active enthusiast of outdoor pursuits. They had been riding a motorbike when they lost their balance and fell, twisting their knee. They heard an audible pop and felt pain in the knee. This was followed by large swelling of the knee within hours of the injury. They visited a local clinic and were investigated and advised rest. A radiograph at this stage demonstrated no suspected bony injury. However, they struggled to mobilize their effected knee in the coming weeks. Pain persisted in the medial and posterior parts of the knee and they were unable to fully extend the joint. Of all the ten patients, seven patients presented with relatively early and were operated within the first month of injury. Two cases presented in the second month of the injury and one case was operated in the third month post injury. They had persistent swelling which was aspirated confirming haemarthrosis. All patients were lacking 15 degrees of extension but had full flexion. They had no collateral instability, but anterior drawer test and Lachman's test were positive in keeping with a complete rupture of the ACL. MRI of knee also confirmed that they had acl ligament avulsions and no other ligament injury. In view of their age and activity profile, they were considered as good candidates for arthroscopic ACL repair to aim to restore their knee function. (Figure 2)

Table I: Distribution of the cases in the study

\begin{tabular}{|c|c|c|c|c|c|}
\hline Case no. & Age & Gender & Site Involved & Grade* & Injury Surgery Interval \\
\hline 1 & 20 & M & Left & T - M3B & 21 days \\
\hline 2 & 32 & M & Right & T-M3A & 47 days \\
\hline 3 & 27 & M & Right & F - M1 & 45 days \\
\hline 4 & 28 & M & Right & T-M1 & 6 days \\
\hline 5 & 38 & M & Left & F - M1 & 11 days \\
\hline 6 & 25 & F & Right & F-M3A & 20 days \\
\hline 7 & 20 & F & Right & T-M3B & 16 days \\
\hline 8 & 18 & M & Left & T-M3B & 15 days \\
\hline 9 & 28 & M & Left & T-M3A & 63 days \\
\hline 10 & 17 & M & Right & T-M3B & 19 days \\
\hline
\end{tabular}

*Meyers and McKeevers classification

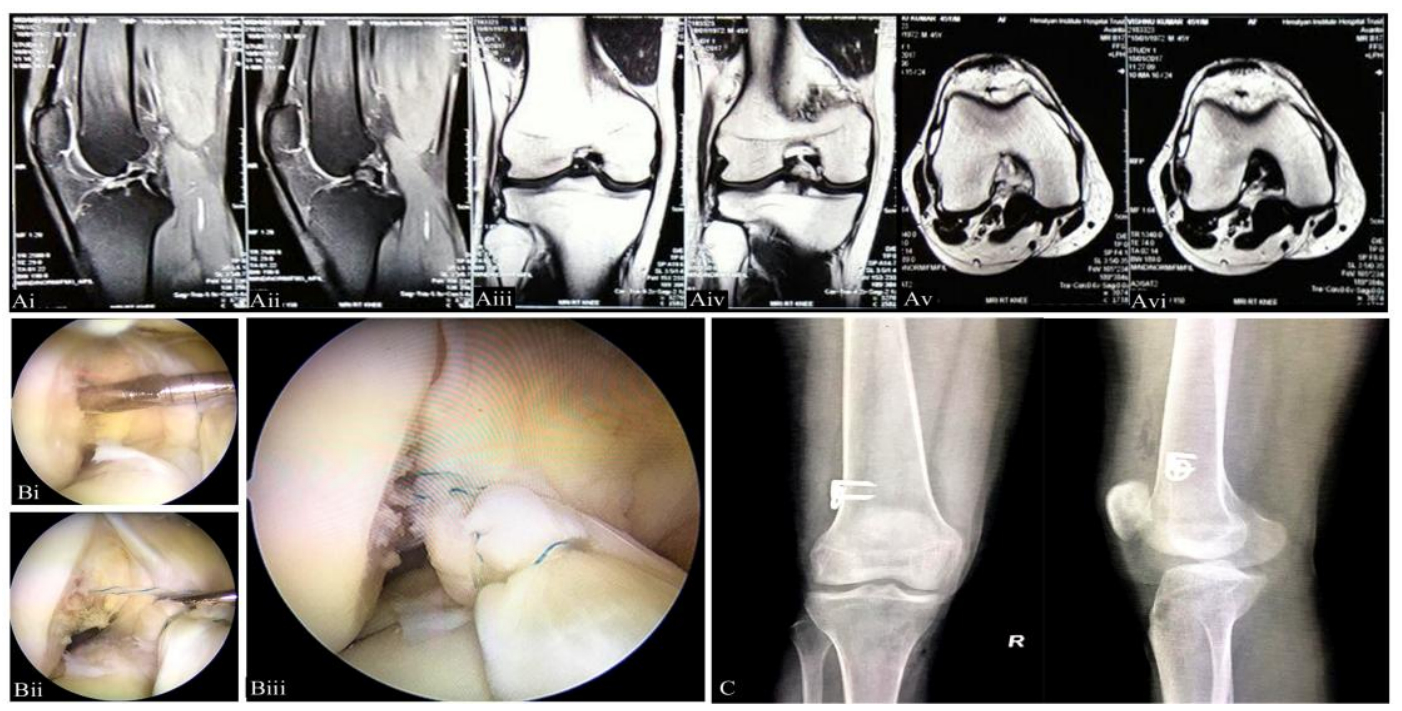

Fig 2: A (i-vi) MRI images showing ACL Avulsion from femoral attachment Right Knee (Shermann Classification Type I). B. Intra operative Images showing (i) $4 \mathrm{~mm}$ drill for making femoral tunnel. (ii) Fibre wire being passed from femoral tunnel (iii) Internally braced ACL being locked through the tunnel at its femoral attachment. C. Post-operative xrays of right knee.

Out of 10 cases three cases had an avulsion from femoral end while seven had it from the tibial end. The injury surgey interval was in a range of six days to sixtythree days. All the cases were operated with the standard repair technique and post-operative regimen was same in all the patients. Post operatively four patients had restriction of knee extension ( 5 degrees) at 4 weeks follow up. Rest of the cases had attained full range of motion at four weeks. Flexion distance was not a difficulty in any of the cases. All the cases had pain free gait pattern but needed aggressive physiotherapy to reinforce the normal gait pattern. There was no clinical ACL laxity in any of the cases, whenever seen at routine follow ups. All the cases subjectively reported a stable knee with full weight bearing from 4 weeks onwards. The rehabilitation program was organized under the guidance of physiotherapist. At the last follow up i.e. minimum of six months, none of the 
patients reported any laxity, stiffness, loss of range of motion. Although the subjects were still not started with sports rehab as per time boundations, they did extremely well with respect to satisfaction with the surgery and achieving a stable, pain free and fully mobile knee. (Figure 3)

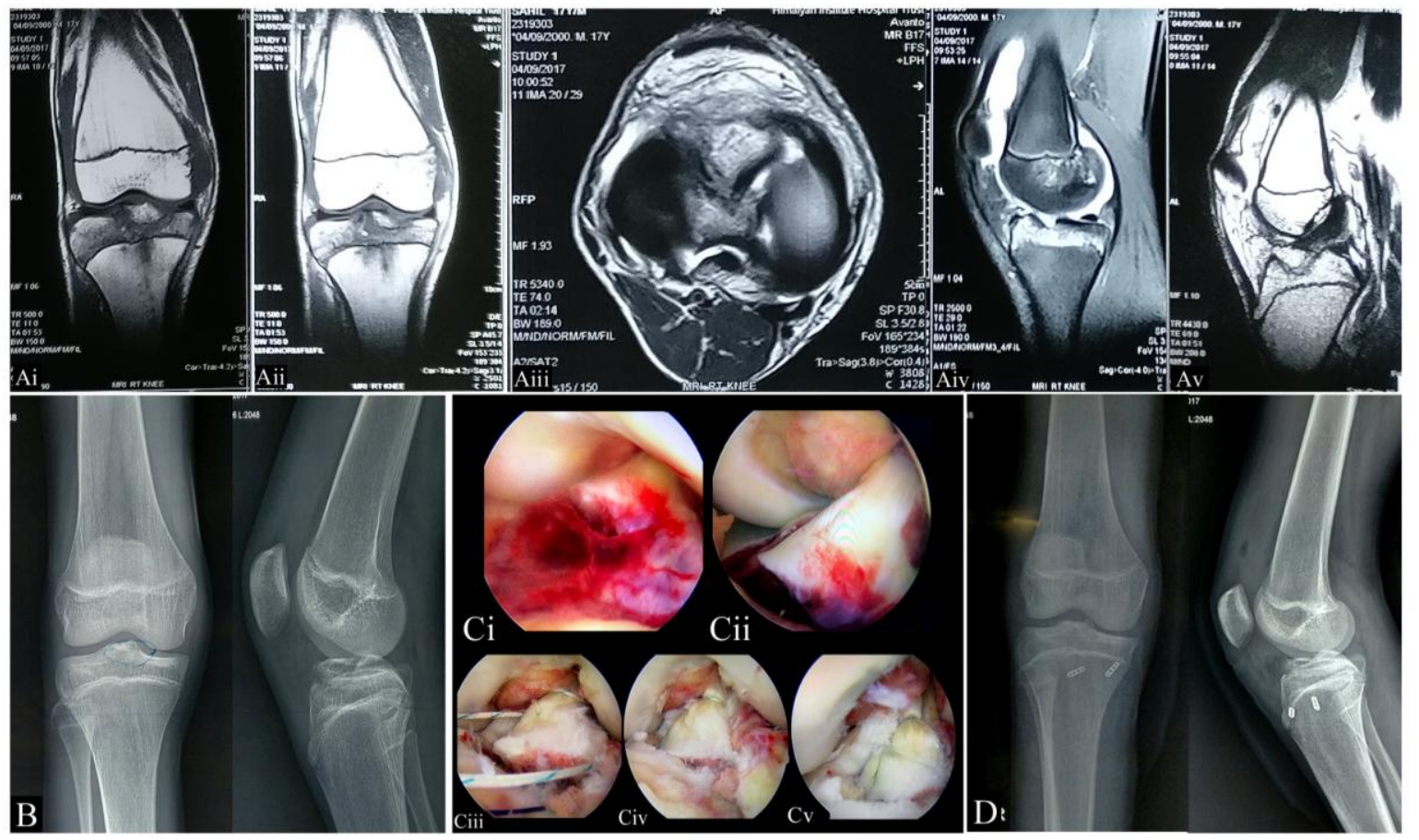

Fig 3: A (i-v): MRI Images showing ACL Avulsion fraction from tibial attachment. B: Pre-operative Xrays; Antero-posterior and lateral views. C Intra-operative images: C (i-ii): Avulsed ACL from tibial attachment. C (iii) Fibre wire being looped around ACL. C (iv-v): internally braced and locked ACL. D: Post-operative Xrays.

\section{Outcome}

The patient's post-operative period was problem-free and they were discharged with an exercise program and weekly physiotherapy sessions. They were reviewed at two weeks, four weeks, 8 weeks, 24 weeks, post operatively. At each stage, they made steady progress with no pain or instability. At eight weeks, their knee was stable and they had a full range of movement with excellent muscle tone. At the last follow up of a minimum of 6 months in all patients, they were back attending their job five times per week and extremely content with their outcome. Though all of them haven't started sports \& recreational activities but the progress has been steady towards a stable and pain free knee with full range of motion.

\section{Discussion}

Primary repair of ACL tears is an age old concept. Most of the arthroscopic surgeons would not agree to a primary repair as the historical results do not favour this procedure. Strand $e t$ al. ${ }^{[12]}$ when reporting there long term follow up results, even opined that "if the same results could be accomplished by a smaller, arthroscopic procedure, primary repair might reduce the number of patients needing later reconstructions with the small costs in the way of risk and inconvenience to the patients," Therefore the research for better fixation methods are justified. Early outcomes with primary repair of the ACL tears have been promising with success rates far exceeding those described historically ${ }^{[13]}$. There have been equal number of reports stating that the primary repair is associated with very high failure rates and less than acceptable results. One of the proposed reasons for these failures could be an overzealous rehabilitation with demands for quick return to sport activities. Learning from these results the rehabilitation in these cases was then slowed down to achieve a better outcome, but the very purpose of surgery became questionable. With further developments the technique of primary ACL repair again regained popularity once the internal fiber ligament bracing technique was introduced. Dynamic Intraligamentary stabilization (DIS) was first described by Eggli et al. ${ }^{[14]}$. It is an operative technique that involves a polyethylene wire passed through the tibial and femoral footprints of the ruptured ACL with a dynamic spring component fixed at the tibial end of the wire. In this technique microfracture is also done at the femoral footprint to enhance the inflammatory healing response. Bodendorfer et al. [15] have reported that according to their results the ideal candidates for this surgery are those with proximal avulsion tears with good tissue quality. MRI gives us a good indication of these potential candidates. They also concluded that the vast majority of patients are not a candidate for repair, but those that are may see better results with this technique. While early results seem promising, long term results are necessary to validate this approach.

The experience of senior authors suggest that residual ACL tissue may persist up until three months from the injury ${ }^{[16]}$. We would agree to these observations that an attempt to primary repair could be successfully attempted till 3 months post injury. Although literature suggest that the key to success in these repairs is to perform it acutely preferably within days $[17,18]$.

One of the limitations of our study is our small sample size. Considering our inclusion criteria \& patients willingness to give consent for this work was a reason for the same. We were also not able to analyze these patients on a functional scale in the post-operative period. This could have drawn better insight into the activity level and patient satisfaction post-surgery. We would recommend further study in the regard so as to gain further knowledge as to how much 
successful a primary repair with ligament augmentation is in the patient rehabilitation back to normalcy.

\section{Conclusion}

The predialect for ACL salvage \& repair is now changing as we have revived \& advanced with technology \& ethical techniques in repairing the torn ligament. The advantage of repairing the ligament over reconstruction is not harvesting the gracilis - semitendinosus hamstring grafts leading to the maintained kinetics \& anatomy of the effected knee function without the hamstring impairment required for harvesting in ACL reconstruction. The internal brace acts as secondary pillar which supports the strength of the repaired ligament. Patients have a better proprioception, faster recovery to work $\&$ is safe guarded against an unpredictable outcome of ACL reconstruction.

\section{References}

1. Feagin JA, Abbott HG, Roukous JA. The isolated tear of the ACL. J Bone Joint Surg Am. 1972; 54(6):1340.

2. Cabaud HE, Feagin JA, Rodkey WG. Acute anterior cruciate ligament injury and augmented repair. Experimental studies. Am J Sports Med. 1980; 8(6):395401.

3. Feagin JA Jr, Curl WW. The Isolated tear of the ACL: 5 year follow up study. Am J Sports Med. 1976; 4(3):95100.

4. Spencer EE, Chissell HR, Spang JT, Feagin JA Jr, Manoff EM, Rohatgi SD. Behavior of sutures used in anterior cruciate ligament reconstructive surgery. Knee Surg Sports Traumatol Arthrosc. 1996; 4(2):84-8.

5. Cabaud HE, Rodkey WG, Feagin JA. Experimental studies of acute anterior cruciate ligament injury and repair. Am J Sports Med. 1979; 7(1):18-22.

6. Robson AW. VI. Ruptured crucial ligaments and their repair by operation. Ann Surg. 1903; 37(5):716-718.

7. Sandberg R, Balkfors B, Nilsson B, et al. Operative versus non-operative treatment of recent injuries to the ligaments of the knee. A prospective randomized study. J Bone Joint Surg Am. 198; 69(8):1120-6.

8. Sherman MF, Lieber L, Bonamo JR, Podesta L, Reiter I. The long-term followup of primary anterior cruciate ligament repair. Defining a rationale for augmentation. Am J Sports Med. 1991; 19(3):243-55.

9. Pai SK, Aslam Pervez N, Radcliffe G. Osteochondral avulsion fracture of the femoral origin of the anterior cruciate ligament in an 11-year-old child. Case Rep Med. 2012; 506798. doi:10.1155/2012/506798

10. Zaricznyj B. Avulsion fracture of the tibial eminence: treatment by open reduction and pinning. J Bone Joint Surg Am. 1978; 59(8):1111-4.

11. Difelice GS, Villegas C, Taylor S. Anterior Criciate Ligament Preservation: Early Results of a Noval Arthroscopic Technique for Suture Anchor Primary Anterior Cruciate Ligament Repair. Arthroscopy. 2015; 31(11):2162-71.

12. Stand T, Molster A, Hordvik M, Krukhaug Y. Long term followup after primary repair of the anterior cruciate ligament: Clinical and Radiological evaluation 15-23 years post operatively. Arch Orthop Trauma Surg. 2005; 125:217-21.

13. Taylor SA, Khair MM, Roberts TR, et al. Primary Repair of the Anterior Cruciate Ligament: A Systematic Review. Arthroscopy. 2015; 31(11):2233-47.

14. Eggli S, Kohlhof H, Zumstein M, Henle P, Hartel M,
Evangelopoulous DS et al. Dynamic Intraligamentous stabilization: novel technique for preserving the ruptured ACL. Knee Surg Sports Traumatol Arthrosc. 2015; 23(4):1215-21.

15. Bodendorfer BM, Hawken JB, Postma WF. Primary Repair of the Anterior Cruciate ligament: A review of the literature. J Orth Rhe Sp Med. 1(3):111.

16. Mackay G, Anthony IC, Jenkins PJ, et al. Anterior Cruciate ligament Repair Revisited. Preliminary Results of Primary Repair with Internal Brace ligament Augmentation: A case Series. Orthop Muscul Syst. 2015; $4: 2$.

17. Warren RF. Primary Repair of the Anterior Cruciate Ligament. Clin Orthop Relat Res. 1983, 65-70.

18. Aho AJ, Lehto MU, Kujala UM. Repair of the Anterior Cruciate Ligament. Augmentation versus Conventional Suture of fresh rupture. Acta Orthop Scand. 1986; 57:354-57. 